\title{
SURFACES WITH ORTHOGONAL FAMILIES OF CIRCLES
}

\author{
THOMAS IVEY
}

(Communicated by Christopher Croke)

\begin{abstract}
The lines of curvature on a cyclide of Dupin are circular arcs. A surface which carries two orthogonal families of circular arcs must arise as an integral surface of an overdetermined exterior differential system. We show that the only solutions of this system are the cyclides of Dupin.
\end{abstract}

\section{INTRODUCTION}

The cyclides of Dupin are a well-known family of surfaces in three-dimensional Euclidean space. A Dupin cyclide can be characterized as the envelope of spheres tangent to three fixed spheres or, locally, as a surface both of whose focal surfaces degenerate to smooth curves. Consequently, the lines of curvature on a cyclide of Dupin are circles. In this article we give a new characterization of these surfaces.

In the course of an explanatory article [P], U. Pinkall conjectured that the cyclides of Dupin are the only surfaces in Euclidean space on which two orthogonal families of circles lie. We confirm this conjecture:

Theorem 1. Let $S$ be a smooth connected surface containing two orthogonal families of circular arcs. Then either the arcs are lines of curvature or they make an angle of $45^{\circ}$ with the lines of curvature, and the lines of curvature are circular arcs.

Recall that the conformal group $O(4,1)$ acts on $S^{3}$ as the projectivized null cone in Lorentzian $\mathbb{R}^{5}$ and on $\mathbb{R}^{3}$ by stereographic projection. Since $O(4,1)$ preserves the set of spheres and planes in $\mathbb{R}^{3}$, it takes circles to circles and hence preserves the set of surfaces carrying two orthogonal families of circular arcs.

Theorem 2. If $S$ contains two orthogonal families of circles that make an angle of $45^{\circ}$ with the lines of curvature, $S$ is equivalent to a Willmore torus (i.e., a torus of revolution in $\mathbb{R}^{3}$ with radii 1 and $\left.\sqrt{2}\right)$ under the action of $O(4,1)$.

In $\S 2$ we review the conformal geometry of curves and surfaces using moving frames, allowing us to show that a surface carrying two orthogonal families of

Received by the editors June 23, 1993.

1991 Mathematics Subject Classification. Primary 53A30; Secondary 58A15.

Key words and phrases. Dupin cyclides, exterior differential systems. 
circles distinct from the lines of curvature corresponds to an integral surface of an overdetermined exterior differential system. In $\S 3$ we show that all integral surfaces of this system must also be integral surfaces of a certain Frobenius system of codimension two on the bundle of conformal frames, thus proving Theorem 1. In $\S 4$ we explicitly integrate this system to prove Theorem 2.

\section{CONFORMAL GEOMETRY VIA MOVING FRAMES}

We begin by setting out explicit formulas for embedding $\mathbb{R}^{3}$ in $S^{3}$ and identifying $S^{3}$ with the lines on the null cone in $\mathbb{R}^{5}$. We will use stereographic projection into $S^{3}$

$$
\left(x_{1}, x_{2}, x_{3}\right) \mapsto\left(\frac{2 x_{1}}{1+R^{2}}, \frac{2 x_{2}}{1+R^{2}}, \frac{2 x_{3}}{1+R^{2}}, \frac{1-R^{2}}{1+R^{2}}\right),
$$

where $R^{2}=x_{1}^{2}+x_{2}^{2}+x_{3}^{2}$, with the inverse map

$$
\left(w_{1}, w_{2}, w_{3}, w_{4}\right) \mapsto\left(\frac{w_{1}}{1+w_{4}}, \frac{w_{2}}{1+w_{4}}, \frac{w_{3}}{1+w_{4}}\right) .
$$

We send $\left(w_{1}, w_{2}, w_{3}, w_{4}\right) \in S^{3}$ to the point in $\mathbb{R P}^{4}$ with homogeneous coordinates $\left[w_{1}, w_{2}, w_{3}, w_{4}, 1\right]$, which lies in the zero locus of the quadratic form $z_{1}^{2}+z_{2}^{2}+z_{3}^{2}+z_{4}^{2}-z_{5}^{2}$. Let $\pi$ denote the projection from $\mathbb{R}^{5}$ minus a hyperplane into $\mathbb{R}^{4}$ that inverts this map:

$$
\pi:\left(z_{1}, z_{2}, z_{3}, z_{4}, z_{5}\right) \mapsto\left(z_{1} / z_{5}, z_{2} / z_{5}, z_{3} / z_{5}, z_{4} / z_{5}\right) .
$$

We study the properties of curves and surfaces in $S^{3}$ invariant under $O(4,1)$ by adapting conformal frames along these objects. A conformal frame will be a basis $\left(e_{0}, e_{1}, e_{2}, e_{3}, e_{4}\right)$ of vectors in $\mathbb{R}^{5}$ whose Lorentzian inner products satisfy

$$
\begin{gathered}
\left\langle e_{i}, e_{j}\right\rangle=\delta_{i j}, \quad\left\langle e_{i}, e_{0}\right\rangle=\left\langle e_{i}, e_{4}\right\rangle=0, \quad 1 \leq i, j \leq 3 \\
\left\langle e_{0}, e_{0}\right\rangle=\left\langle e_{4}, e_{4}\right\rangle=0, \quad\left\langle e_{0}, e_{4}\right\rangle=-1 .
\end{gathered}
$$

Let $\mathscr{F}$ be the manifold consisting of all such frames. On $\mathscr{F}$ we define 1 -forms $\omega_{\beta}^{\alpha}$ by

$$
d e_{\alpha}=e_{\beta} \omega_{\alpha}^{\beta}, \quad 0 \leq \alpha, \beta \leq 4 .
$$

It follows that $d \omega_{\beta}^{\alpha}=-\omega_{\gamma}^{\alpha} \wedge \omega_{\beta}^{\gamma}$ and that

$$
\begin{aligned}
& \omega_{j}^{i}=-\omega_{i}^{j}, \\
& \omega_{i}^{4}=\omega_{0}^{i}, \omega_{4}^{i}=\omega_{i}^{0}, \\
& \omega_{4}^{0}=\omega_{0}^{4}=0, \omega_{4}^{4}=-\omega_{0}^{0} .
\end{aligned}
$$

(Since $O(4,1)$ acts simply transitively on these frames, $\mathscr{F}$ is diffeomorphic to that group, and the $\omega_{\beta}^{\alpha}$ are identified with the left-invariant 1 -forms. This identification will be used in proving Theorem 2 .)

Since $e_{0}$ is a null vector, $\rho:\left(e_{0}, e_{1}, e_{2}, e_{3}, e_{4}\right) \mapsto \pi\left(e_{0}\right)$ gives a submersion to $S^{3}$. In fact, $\mathscr{F}$ is a principal bundle over $S^{3}$, with seven-dimensional fibre, via this submersion. Equation (2) shows that the forms $\omega_{0}^{1}, \omega_{0}^{2}, \omega_{0}^{3}$ are semi-basic on $\mathscr{F}$, i.e., they annihilate vertical vectors. We will use the slightly abbreviated notation $\omega^{1}, \omega^{2}, \omega^{3}$ for these forms. 
Let $\gamma: I \rightarrow S^{3}$ be an immersed curve ( $I$ is some open interval). A conformal framing along $\gamma$ is any smooth lifting into $\mathscr{F}$ :

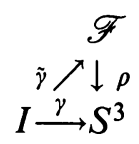

By a 1-adapted framing we will mean one such that, when pulled back to $I$, $d e_{0}=e_{0} \omega_{0}^{0}+e_{1} \omega^{1}$. (Equivalently, $\tilde{\gamma}^{*} \omega_{0}^{2}=\tilde{\gamma}^{*} \omega_{0}^{3}=0$.) It follows that for a 1-adapted framing along an immersed curve, $\tilde{\gamma}^{*} \omega^{1} \neq 0$. (Note that we can always modify an arbitrary framing to get a 1-adapted framing by using the $O(3)$ action on the vectors $e_{1}, e_{2}, e_{3}$.)

In general, $d e_{1}=e_{0} \omega_{1}^{0}+e_{2} \omega_{1}^{2}+e_{3} \omega_{1}^{3}+e_{4} \omega^{1}$. However, we can make the following modifications to a conformal frame while preserving 1-adaptedness:

$$
\begin{aligned}
& \bar{e}_{2}=e_{2}+\xi_{2} e_{0}, \quad \xi_{2}, \xi_{3} \in \mathbb{R}, \\
& \bar{e}_{3}=e_{3}+\xi_{3} e_{0}, \\
& \bar{e}_{4}=e_{4}+\xi_{2} e_{2}+\xi_{3} e_{3}+\frac{1}{2}\left(\xi_{2}^{2}+\xi_{3}^{2}\right) e_{0} .
\end{aligned}
$$

Since $\tilde{\gamma}^{*} \omega^{1}$ is nowhere zero, we can arrange that $d e_{1}=e_{0} \omega_{1}^{0}+e_{4} \omega^{1}$ along $\tilde{\gamma}$ (i.e., $\left.\tilde{\gamma}^{*} \omega_{1}^{2}=\tilde{\gamma}^{*} \omega_{1}^{3}=0\right)$. Call this a 2-adapted framing.

Now assume that $e_{0}, e_{1}, e_{4}$ are part of a 2-adapted framing along the curve. Since the plane spanned by $e_{0}$ and $e_{1}$ projects down (via $\pi$ ) to the tangent line to the curve in $S^{3}$, we will say that $e_{0} \wedge e_{1}$ represents the tangent 2-plane to the curve. Since $d\left(e_{0} \wedge e_{1}\right)=e_{0} \wedge e_{1} \omega_{0}^{0}+e_{0} \wedge e_{4} \omega^{1}$, we will say that $e_{0} \wedge e_{1} \wedge e_{4}$ represents the osculating 3-plane. (It is easy to check that these objects are well defined.) If $e_{0} \wedge e_{1} \wedge e_{4}$ is fixed up to multiple along $\gamma$, then $\pi\left(e_{0}\right)$ lies in a fixed two-dimensional affine subspace of $\mathbb{R}^{4}$. It is easy to see that this means the image of $\gamma$ is the stereographic projection of a circle or a straight line in $\mathbb{R}^{3}$. Since this conformal characterization of circles is enough for our purposes, we will move on to surfaces.

Let $\Sigma^{2} \stackrel{\phi}{\rightarrow} S^{3}$ be an immersed surface, and let $\tilde{\phi}$ be a conformal framing defined on an open set $U \in \Sigma^{2}$. We can use the $O(3)$ action to arrange that

$$
d e_{0}=e_{0} \omega_{0}^{0}+e_{1} \omega^{1}+e_{2} \omega^{2}
$$

on $U$, i.e., that $\tilde{\phi}^{*} \omega_{0}^{3}=0$. Call such a framing 1-adapted. It will be useful to consider the bundle $\mathscr{F}_{1}(\phi)$ over $\Sigma^{2}$ consisting of all 1-adapted frames for the immersion $\phi$. Its fibre is five-dimensional, since in addition to acting on the pair $e_{1}, e_{2}$ by $O(2)$, the following modifications preserve "1-adaptedness":

$$
\begin{aligned}
& \bar{e}_{0}=\alpha e_{0}, \quad \alpha \neq 0, \\
& \bar{e}_{1}=e_{1}+\xi_{1} e_{0}, \quad \xi_{1}, \xi_{2}, \xi_{3} \in \mathbb{R}, \\
& \bar{e}_{2}=e_{2}+\xi_{2} e_{0}, \\
& \bar{e}_{3}=e_{3}+\xi_{3} e_{0}, \\
& \bar{e}_{4}=\frac{1}{\alpha}\left(e_{4}+\xi_{1} e_{1}+\xi_{2} e_{2}+\xi_{3} e_{3}+\frac{1}{2}\left(\xi_{1}^{2}+\xi_{2}^{2}+\xi_{3}^{2}\right) e_{0}\right) .
\end{aligned}
$$

While $\omega^{1}, \omega^{2}$ are semibasic for $\mathscr{F}_{1}(\phi)$, this action shows that the forms $\omega_{1}^{2}$, $\omega_{0}^{0}, \omega_{1}^{0}, \omega_{2}^{0}, \omega_{3}^{0}$ form a coframe when restricted to a fibre of $\mathscr{F}_{1}(\phi)$. 
The equations $\omega^{3}=0$ and $d \omega^{3}=0$ imply that there are smooth functions $a, b, c$ on $\mathscr{F}_{1}(\phi)$ such that

$$
\begin{aligned}
& \omega_{1}^{3}=a \omega^{1}+b \omega^{2}, \\
& \omega_{2}^{3}=b \omega^{1}+c \omega^{2} .
\end{aligned}
$$

We can ascertain how $a, b, c$ vary along the fibres by differentiating:

$$
\left.\begin{array}{rl}
d a & \equiv-a \omega_{0}^{0}+2 b \omega_{1}^{2}+\omega_{3}^{0} \\
d b & \equiv-b \omega_{0}^{0}+(a-c) \omega_{2}^{1} \\
d c & \equiv-c \omega_{0}^{0}-2 b \omega_{1}^{2}+\omega_{3}^{0}
\end{array}\right\} \bmod \omega^{1}, \omega^{2} .
$$

In particular, $d(a+c) \equiv-(a+c) \omega_{0}^{0}+2 \omega_{3}^{0}$ shows that we can arrange that $a+c=0$ by moving along the fibres, and

$$
d\left(\begin{array}{c}
a-c \\
2 b
\end{array}\right) \equiv-\left(\begin{array}{cc}
\omega_{0}^{0} & 2 \omega_{2}^{1} \\
2 \omega_{1}^{2} & \omega_{0}^{0}
\end{array}\right)\left(\begin{array}{c}
a-c \\
2 b
\end{array}\right) \bmod \omega^{1}, \omega^{2}
$$

shows that the group $C O(2)$ acts on the remaining variables. It follows that the quadratic form

$$
\mathscr{Q}=b\left(\left(\omega^{2}\right)^{2}-\left(\omega^{1}\right)^{2}\right)+(a-c) \omega^{1} \omega^{2}
$$

is well defined, up to multiple, on $\Sigma^{2}$. In fact, the null directions of $\mathscr{Q}$ are principal directions on the surface. For, suppose that $e_{1}$ points along a null curve for $\mathscr{Q}$ and $t$ is a parameter along such a curve. This implies that $b=0$ along the curve, and since $d e_{2} \equiv e_{0} \omega_{2}^{0}+e_{1} \omega_{2}^{1}+e_{3}\left(b \omega^{1}\right) \bmod \omega^{2}$, there is no component of $d\left(\pi_{*} e_{2}\right) / d t$ in the direction of $\pi_{*} e_{3}$, and the geodesic torsion is zero. So $\mathscr{Q}$ is nothing but the conformal version of the third fundamental form of $\Sigma^{2}$.

Now assume that $\phi\left(\Sigma^{2}\right)$ carries two distinguished orthogonal families of curves. On an open set $U$ we can get a 1-adapted framing where $e_{1}$ and $e_{2}$ point along the curves in the respective families. If on $U$ these directions do not coincide with the principal directions, then $b \neq 0$ and we can arrange that $a+c=0$ and $b=1$ at each point. Let $\mathscr{F}_{2}(\phi)$ be the subbundle of adapted frames, tangent to the two families of curves, and for which $a+c=0$ and $b=1$. Since we can no longer rotate $e_{1}$ and $e_{2}, \omega_{1}^{2}$ is semibasic on $\mathscr{F}_{2}(\phi)$, and because of (4), $\omega_{0}^{0}$ and $\omega_{3}^{0}$ are also semibasic. (However, the fibres are two-dimensional since we may still add multiples of $e_{0}$ to $e_{1}$ and $e_{2}$; this shows that $\omega_{1}^{0}$ and $\omega_{2}^{0}$ form a coframe along the fibres of $\mathscr{F}_{2}(\phi)$.) There will be smooth functions $t_{1}, t_{2}, u_{1}$, and $u_{2}$ on $\mathscr{F}_{2}(\phi)$ such that

$$
\begin{aligned}
& \omega_{1}^{2}=t_{1} \omega^{1}+t_{2} \omega^{2}, \\
& \omega_{0}^{0}=u_{1} \omega^{1}+u_{2} \omega^{2} .
\end{aligned}
$$

Differentiation shows that $d u_{i} \equiv-\omega_{i}^{0} \bmod \omega^{1}, \omega^{2}$. So by moving along the fibers of $\mathscr{F}_{2}(\phi)$ we can always get a framing such that $u_{1}=u_{2}=0$ along $\tilde{\phi}\left(\Sigma^{2}\right)$.

Assume $\tilde{\phi}$ is such a framing. Since $e_{0} \wedge e_{1}$ is the tangent 2-plane to the $e_{1}$-curves,

$$
d\left(e_{0} \bigwedge e_{1}\right) \equiv e_{0} \bigwedge\left(t_{1} e_{2}+a e_{3}+e_{4}\right) \omega^{1} \bmod \omega^{2}
$$

shows that the osculating 3-plane for this curve is $e_{0} \wedge e_{1} \wedge\left(t_{1} e_{2}+a e_{3}+e_{4}\right)$. Similarly, the osculating 3-plane for an $e_{2}$-curve is $e_{0} \wedge e_{2} \Lambda\left(-t_{2} e_{1}-a e_{3}+e_{4}\right)$. 
If the $e_{1}$-and $e_{2}$-curves are assumed to be circles, the osculating 3-planes should be fixed along the respective curves. Differentiating the above 3-vectors, using the structure equations (2), leads us to the following

Proposition. Suppose $\tilde{\phi}$ is a conformal framing, adapted in the above manner, along a surface $\Sigma^{2}$ carrying two orthogonal families of circles which are not lines of curvature. Then $\tilde{\phi}\left(\Sigma^{2}\right)$ is an integral surface of the following differential forms on $\mathscr{F} \times \mathbb{R}^{3}$, where we use $a, t_{1}$, and $t_{2}$ as coordinates on the $\mathbb{R}^{3}$ factor:

$$
\begin{array}{ll}
\theta_{0}=\omega^{3} & \left(d a+t_{1} \omega^{1}+\omega_{3}^{0}\right) \wedge \omega^{2} \\
\theta_{1}=\omega_{1}^{3}-\left(a \omega^{1}+\omega^{2}\right) & \left(d a+t_{2} \omega^{2}-\omega_{3}^{0}\right) \wedge \omega^{1} \\
\theta_{2}=\omega_{2}^{3}-\left(\omega^{1}-a \omega^{2}\right) & \left(d t_{1}-a \omega^{1}+\omega_{2}^{0}\right) \wedge \omega^{2} \\
\theta_{3}=\omega_{1}^{2}-\left(t_{1} \omega^{1}+t_{2} \omega^{2}\right) & \left(d t_{2}-a \omega^{2}-\omega_{1}^{0}\right) \wedge \omega^{1} . \\
\theta_{4}=\omega_{0}^{0} &
\end{array}
$$

In other words, these forms vanish when restricted to $\tilde{\phi}\left(\Sigma^{2}\right)$. We also note that $\omega_{1} \wedge \omega_{2}$ restricts to be a nowhere-vanishing 2-form on $\tilde{\phi}\left(\Sigma^{2}\right)$.

\section{THE EXTERIOR DIFFERENTIAL SYSTEM}

An integral surface of a 1-form $\theta$ is also an integral of $d \theta$. So we need to calculate the exterior derivatives of the above 1-forms:

$$
\left.\begin{array}{rl}
d \theta_{0} & \equiv 0 \\
d \theta_{1} & \equiv-\left(d a+\omega_{3}^{0}-2\left(a t_{1}+t_{2}\right) \omega^{2}\right) \wedge \omega^{1} \\
d \theta_{2} \equiv & \left(d a-\omega_{3}^{0}-2\left(t_{1}-a t_{2}\right) \omega^{1}\right) \wedge \omega^{2} \\
d \theta_{3} \equiv & -\left(d t_{1}+\omega_{2}^{0}\right) \wedge \omega^{1}-\left(d t_{2}-\omega_{1}^{0}\right) \wedge \omega^{2} \\
& +\left(1+a^{2}-t_{1}^{2}-t_{2}^{2}\right) \omega^{1} \wedge \omega^{2} \\
d \theta_{4} \equiv & -\omega_{1}^{0} \wedge \omega^{1}-\omega_{2}^{0} \wedge \omega^{2}
\end{array}\right\} \bmod \theta_{0}, \theta_{1}, \theta_{2}, \theta_{3}, \theta_{4} .
$$

These 2-forms, together with the forms given in the above proposition, generate an exterior ideal $\mathscr{J}_{0}$ that is closed under differentiation. However, this exterior differential system is not involutive since the values of $d a$ and $\omega_{3}^{0}$ on an integral surface turn out to be uniquely determined by the vanishing of the 2-forms (see [EDS, IV.1.8] for a simple example of how this implies noninvolutivity). In fact, on any integral surface the following 1 -forms must vanish:

$$
\begin{aligned}
& \theta_{5}=\left(t_{1}-2 a t_{2}\right) \omega^{1}+\left(t_{2}+2 a t_{1}\right) \omega^{2}-2 d a, \\
& \theta_{6}=\left(2 a t_{2}-3 t_{1}\right) \omega^{1}+\left(2 a t_{1}+3 t_{2}\right) \omega^{2}-2 \omega_{3}^{0} .
\end{aligned}
$$

Thus, our surface is an integral of a larger exterior differential system $\mathscr{I}_{1}$, obtained from $\mathscr{I}_{0}$ by adding $\theta_{5}$ and $\theta_{6}$ and their exterior derivatives modulo $\theta_{0} \cdots \theta_{6}$ :

$$
\begin{aligned}
d \theta_{5} \equiv & \left(d t_{1}-2 a d t_{2}\right) \wedge \omega^{1}+\left(d t_{2}+2 a d t_{1}\right) \wedge \omega^{2}+2\left(t_{1}^{2}+t_{2}^{2}\right) \omega^{1} \wedge \omega^{2} \\
d \theta_{6} \equiv & \left(2 a d t_{2}-3 d t_{1}-2 a \omega_{1}^{0}-2 \omega_{2}^{0}\right) \wedge \omega^{1} \\
& +\left(2 a d t_{1}+3 d t_{2}-2 \omega_{1}^{0}+2 a \omega_{2}^{0}\right) \wedge \omega^{2}+2\left(t_{2}^{2}-t_{1}^{2}\right) \omega^{1} \wedge \omega^{2}
\end{aligned}
$$

However, $\mathscr{I}_{1}$ is not involutive: its Cartan characters are $s_{1}=4, s_{2}=0$, while the vanishing of the 2 -forms determines, up to only $t w o$ parameters at each point, the values of $d t_{1}, d t_{2}, \omega_{1}^{0}$, and $\omega_{2}^{0}$. (The reader is again referred to 
[EDS] for Cartan's test for involutivity.) In fact, there must be smooth functions $u$ and $v$ such that the following 1-forms vanish:

$$
\begin{aligned}
\theta_{7} & =2 \omega_{1}^{0}+\left(6 u+1+5 a^{2}+3 t_{2}^{2}-t_{1}^{2}\right) \omega^{1}+v \omega^{2}, \\
\theta_{8} & =2 \omega_{2}^{0}+\left(-6 u+1+5 a^{2}+3 t_{1}^{2}-t_{2}^{2}\right) \omega^{2}+v \omega^{1}, \\
\theta_{9} & =d t_{1}-(a+v / 2) \omega^{1}+2\left(u-a^{2}-t_{1}^{2}\right) \omega^{2}, \\
\theta_{10} & =d t_{2}-(a-v / 2) \omega^{2}+2\left(u+a^{2}+t_{2}^{2}\right) \omega^{1} .
\end{aligned}
$$

We can now think of the surface as an integral of a Pfaffian system $\mathscr{I}_{2}$ on $\mathscr{F} \times \mathbb{R}^{5}$, where we introduce $u$ and $v$ as new variables, generated by $\theta_{0} \cdots \theta_{10}$ and their exterior derivatives. (The 2-forms listed in the above proposition, which are not themselves exterior derivatives, are now congruent to zero modulo $\theta_{5} \cdots \theta_{10}$.) The exterior derivatives of $\theta_{7} \cdots \theta_{10}$, modulo the 1-forms, are 2forms whose vanishing uniquely determines the values of $d u$ and $d v$; in fact, the 1 -forms

$$
\begin{aligned}
\theta_{11}= & d u+\left(2 u t_{2}+a t_{1}-2 t_{2}\right) \omega^{1}-\left(2 u t_{1}+a t_{2}+2 t_{1}\right) \omega^{2}, \\
\theta_{12}= & d v+\left(5 v t_{2}-4 u t_{1}+4 t_{1}\left(t_{2}^{2}-a^{2}\right)-16 a t_{2}-9 t_{1}\right) \omega^{1} \\
& -\left(5 v t_{1}+4 u t_{2}+4 t_{2}\left(t_{1}^{2}-a^{2}\right)+16 a t_{1}+9 t_{2}\right) \omega^{2}
\end{aligned}
$$

must vanish. Differentiating these forms modulo $\theta_{0} \cdots \theta_{12}$ gives

$$
\begin{aligned}
& d \theta_{11} \equiv-\left(2 v+13 t_{1} t_{2}\right) \omega^{1} \wedge \omega^{2} \\
& d \theta_{12} \equiv\left(-36 u+36 t_{2}^{2}-36 t_{1}^{2}-26 a v-160 a t_{1} t_{2}\right) \omega^{1} \wedge \omega^{2}
\end{aligned}
$$

Thus, on any integral surface of $\mathscr{I}_{2}$ where $\omega^{1} \wedge \omega^{2} \neq 0$,

$$
\begin{aligned}
& u=\frac{a t_{1} t_{2}}{4}-t_{1}^{2}+t_{2}^{2}, \\
& v=-\frac{13}{2} t_{1} t_{2} .
\end{aligned}
$$

Finally, we substitute these expressions into $\theta_{11}$ and $\theta_{12}$ and use the known values of $d a, d t_{1}$, and $d t_{2}$ to obtain two linear combinations of $\omega^{1}$ and $\omega^{2}$ that must vanish along the integral surface. Their coefficients are the polynomials

$$
\begin{gathered}
P_{1}=117 t_{1} t_{2}^{2}-72 t_{1}+72 t_{1} a^{2}-72 t_{1}^{3}+18 t_{1}^{2} a t_{2}-180 a t_{2}, \\
P_{2}=-180 a t_{1}-72 a^{2} t_{2}+72 t_{2}^{3}-117 t_{2} t_{1}^{2}+18 t_{1} a t_{2}^{2}+72 t_{2}, \\
P_{3}=-16 a t_{1}-96 t_{2}^{3}+138 t_{2} t_{1}^{2}-41 t_{1} a t_{2}^{2}-32 t_{2}-60 a^{2} t_{2}+8 a t_{1}^{3}-2 a^{2} t_{1}^{2} t_{2}-8 a^{3} t_{1}, \\
P_{4}=138 t_{1} t_{2}^{2}-32 t_{1}-96 t_{1}^{3}+41 t_{1}^{2} a t_{2}+16 a t_{2}-60 t_{1} a^{2}-8 a t_{2}^{3}-2 a^{2} t_{2}^{2} t_{1}+8 a^{3} t_{2} .
\end{gathered}
$$

It follows that any integral surface of $\mathscr{I}_{2}$ must lie inside the common zero locus of these polynomials; in order to classify surfaces with orthogonal families of circles, we must study the real points of this locus.

First of all, these polynomials vanish when $t_{1}=t_{2}=0$. If this is the case on an open subset of $\Sigma^{2}$, then $u=v=0$ and $\theta_{9}=\theta_{10}=0$ imply that $a=0$. It then follows that $\tilde{\phi}\left(\Sigma^{2}\right)$ is an integral of the Pfaffian system

$$
\mathcal{J}=\left\{\omega^{3}, \omega_{1}^{3}-\omega^{2}, \omega_{2}^{3}-\omega^{1}, \omega_{1}^{2}, \omega_{0}^{0}, 2 \omega_{1}^{0}+\omega^{1}, 2 \omega_{2}^{0}+\omega^{2}, \omega_{3}^{0}\right\},
$$

which, we should note, is a Frobenius system on $\mathscr{F} 10$. On integrals of $\mathscr{J}$, the third fundamental form is $\mathscr{Q}=\left(\omega^{2}\right)^{2}-\left(\omega^{1}\right)^{2}$, so the vectors $e_{1} \pm e_{2}$ point 
in the principal directions. To see that the lines of curvature must, in fact, be circles, we need to calculate the osculating 3-planes for these directions:

$$
d\left(e_{0} \bigwedge\left(e_{1} \pm e_{2}\right)\right) \equiv\left(e_{0} \bigwedge\left(e_{3} \pm e_{4}\right)\right)\left(\omega^{1} \pm \omega^{2}\right) \quad \bmod \mathscr{J}, \omega^{1} \mp \omega^{2}
$$

So, $e_{0} \bigwedge\left(e_{1} \pm e_{2}\right) \bigwedge\left(e_{3} \pm e_{4}\right)$ is the osculating 3-plane in the $e_{1} \pm e_{2}$ principal direction, and it is easy to see that this is fixed along one of the corresponding lines of curvature.

To conclude the proof of Theorem 1, it will suffice to show that there are no other real zeros of the polynomials $P_{1}$ through $P_{4}$. Let $R_{i j}$ be the resultant of $P_{i}$ and $P_{j}$ with respect to $a$, which also must vanish along the common zero locus. If we let $x=t_{1}^{2}$ and $y=t_{2}^{2}$,

$$
R_{12}=100(5 x y-4 x-4 y)\left(x y^{2}+y x^{2}+100\left(x^{2}+y^{2}+x+y\right)-240 x y\right) .
$$

This shows that $t_{1}=0$ if and only if $t_{2}=0$. If neither are zero at some point, then

$$
\begin{aligned}
\frac{R_{24}}{2 t_{2}}-\frac{R_{13}}{2 t_{1}}=(x-y) & \left(15 x^{2} y^{2}+506\left(x^{2} y+y^{2} x\right)\right. \\
+ & \left.6072\left(x^{2}+y^{2}\right)+644 x y+2072(x+y)\right) .
\end{aligned}
$$

Substituting $y=x$ into $R_{12}$ gives two positive roots for $x$, neither of which satisfy $R_{34}=0$. Taking the resultant of $15 x^{2} y^{2}+\cdots+2072(x+y)$ with $5 x y-4 x-4 y$ in $y$ yields a polynomial in $x$ which has only imaginary roots. Taking the resultant with the other factor of $R_{12}$ yields a polynomial in $x$ that has no positive real roots.

\section{EXPLICIT INTEGRATION}

In the previous section we arrived at a Frobenius system $\mathcal{J}$ on the conformal frame bundle $\mathscr{F}$ such that an adapted framing along a surface foliated by orthogonal families of circles distinct from the lines of curvature had to be an integral of $\mathscr{J}$. In this section we will explicitly integrate the Frobenius system $\mathscr{J}$ by exploiting the fact that $\mathscr{F}$ is a Lie group.

The forms $\omega_{\beta}^{\alpha}$ on $\mathscr{F}$ obey the same structure equations as the left-invariant forms on a subgroup of $\mathrm{GL}(5, \mathbb{R})$ and take value in the subalgebra of matrices $A$ such that $J A+{ }^{t} A J=0$ for

$$
J=\left(\begin{array}{ccc}
0 & 0 & -1 \\
0 & I_{3} & 0 \\
-1 & 0 & 0
\end{array}\right)
$$

The corresponding Lie group consists of matrices $M$ such that ${ }^{t} M J M=J$. It is clear that this is the subgroup $G$ of $\operatorname{GL}(5, \mathbb{R})$ that preserves the quadratic form $Q_{2}=z_{2}^{2}+z_{3}^{2}+z_{4}^{2}-2 z_{1} z_{5}$. Since this is equivalent to the quadratic form $Q_{1}=z_{1}^{2}+z_{2}^{2}+z_{3}^{2}+z_{4}^{2}-z_{5}^{2}$ by a linear transformation, $G$ is conjugate to $O(4,1)$ as we usually think of it. If $($,$) denotes the symmetric bilinear form$ corresponding to $Q_{2}$ and $e_{0} \cdots e_{4}$ are the columns of $M$, then

$$
\begin{gathered}
\left(e_{i}, e_{j}\right)=\delta_{i j}, \quad\left(e_{i}, e_{0}\right)=\left(e_{i}, e_{4}\right)=0, \quad 1 \leq i, j \leq 3, \\
\left(e_{0}, e_{0}\right)=\left(e_{4}, e_{4}\right)=0, \quad\left(e_{0}, e_{4}\right)=-1 .
\end{gathered}
$$


This shows that $G$ is diffeomorphic to $\mathscr{F}$, and (2) shows that the 1-forms $\omega_{\beta}^{\alpha}$ form the matrix $g^{-1} d g$ of left-invariant forms on $G$.

Since $\mathcal{J}$ is spanned by left-invariant forms and is Frobenius, its integral surfaces are the left cosets of a Lie subgroup of $G$. The two-dimensional subspace at the identity annihilated by $\mathcal{J}$ is

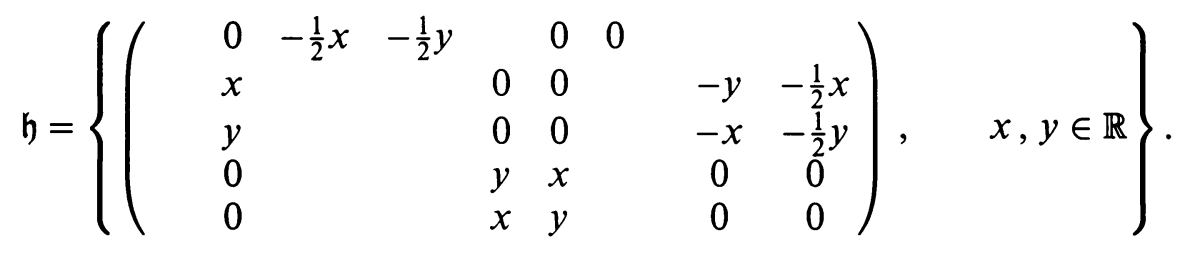

It is easy to check that $\mathfrak{h}$ is a maximal abelian subalgebra. Exponentiating it gives a compact torus in $G$. Our convention in $\S 2$ was that the vector $e_{0}$ traces out the surface in the projectivized null cone in $P\left(\mathbb{R}^{5}\right)$. The first column of the image of $\mathfrak{h}$ under the exponential map is the set of vectors of the form

(5) $e_{0}={ }^{t}\left(\frac{1}{2}(1+\cos x \cos y), \sin x \cos y, \cos x \sin y, \sin x \sin y, 1-\cos x \cos y\right)$.

Since $G$ acts transitively on the left cosets of $\exp (\mathfrak{h})$ and this action covers the action of $G$ on $\mathbb{R}^{5}$ via the map $e_{0}$, all surfaces of the kind in which we are interested will be equivalent under the conformal group to the one parametrized by (5).

It is easy to check that this surface lies in the zero locus of $Q_{2}$ and also of $z_{2} z_{3}-z_{4}\left(z_{1}-\frac{1}{2} z_{5}\right)$. Since in $\S 2$ we use an identification of $S^{3}$ with the null cone of $Q_{1}$, not $Q_{2}$, we need to apply a linear transformation that takes $Q_{2}$ to $Q_{1}$. The resulting surface then lies in the zero locus of $Q_{1}$ and of $2 \sqrt{2} z_{2} z_{3}-z_{4}\left(z_{5}-3 z_{1}\right)$. We can use transformations in $O(4,1)$ to change the latter quadric to $\sqrt{2}\left(z_{1}^{2}+z_{2}^{2}-z_{3}^{2}-z_{4}^{2}\right)$. The resulting surface is in the zero locus of $Q_{1}$ and of $z_{1}^{2}+z_{2}^{2}-z_{3}^{2}-z_{4}^{2}$. If we now take the cone of lines through the origin of $\mathbb{R}^{5}$ to points on this surface, this gives the surface in $S^{3}$ whose points satisfy $w_{1}^{2}+w_{2}^{2}=w_{3}^{2}+w_{4}^{2}$. The image surface in $\mathbb{R}^{3}$ under stereographic projection satisfies $8\left(x_{1}^{2}+x_{2}^{2}\right)=\left(1+x_{1}^{2}+x_{2}^{2}+x_{3}^{2}\right)^{2}$, which is the equation of a torus of revolution about the $x_{3}$-axis, with radii 1 and $\sqrt{2}$.

\section{ACKNOWLEDGMENTS}

Some of the computations discussed here were carried out using Maple V with an improved differential forms package written by Yunliang Yu. The author is grateful to Dr. Yu and to Jay Fillmore for helpful discussions.

\section{REFERENCES}

[EDS] R. L. Bryant, S.-S. Chern, R. B. Gardner, H. Goldschmidt, and P. A. Griffiths, Exterior differential systems, Math. Sci. Res. Inst. Publ., Springer-Verlag, New York, 1990.

[P] U. Pinkall, Cyclides of Dupin, Mathematical Models From the Collections of Universities and Museums, Vol. 2 (Commentary) (G. Fischer, ed.), Vieweg, 1986, pp. 28-30.

Department of Mathematics, University of California San Diego, la Jolla, CaliforNIA 92093-0112

Current address: Department of Mathematics, Case Western Reserve University, 10900 Euclid Ave., Cleveland, Ohio 